\title{
The Importance of Telemedicine in a Post COVID World
}

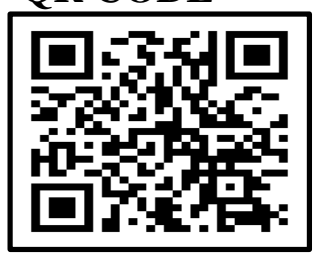

\section{BHUPESH SHARMA ${ }^{1}$, SANJANA MANOCHA*1, NYAGAM YANGZOM²}

Telemedicine has found immense popularity, especially in a COVID-19 affected world. Its application shall improve the quality of health care provided to patients and help in time management as it help facilitates optimized referral systems which are based on the immediate/follow up needs of the patient, decision-making, reduction in patient's waiting time as well as consultation time. This review is an effort to shed light on the main practical applications of telemedicine for emphasizing potentialities, problems, and applications of this relatively new branch in medicine. The paper also highlights the various aspects of telemedicine in relation to the healthcare sector, its advantages/disadvantages in a post COVID-19 world.

KEYWORDS: Telemedicine, Artificial Intelligence, Technology

\section{INTRODUCTION}

The healthcare sector in an ever-evolving branch. A particular advancement in this sector is the "telemedicine", which has help facilitate immediate/short-term and long-term care of the patient. An advantage of this is that health information is communicate between practitioners and the patient through e-mail and interactive chats or videoconferences, which increasing convenience for the patient and reduces the need to physically visit the doctor's clinic. This is especially helpful in remote areas where provision of immediate and urgent care would not be possible. ${ }^{1}$

The use of telemedicine is believed to improve the quality of health care provided to patients and help in time management as it help facilitates optimized referral systems which are based on the immediate/follow up needs of the patient, decisionmaking, reduction in patient's waiting time as well as consultation time. Another added advantage is that telemedicine can be used for peer education and consultations, case discussions among their peers and colleagues. ${ }^{2}$

The entire process of networking, sharing digital information, distant consultations, workup, and analysis is dealt with by a segment of the science of telemedicine and this review paper aims to highlight the various aspects of telemedicine in relation to the healthcare sector, its advantages/disadvantages in a post COVID-19 world. .3

\section{HISTORY}

The first published instance documenting the use of telemedicine occurred in the early $20^{\text {th }}$ century when electrocardiograph data were transmitted through telephone wires. ${ }^{5}$ In its modern from, telemedicine started in the $1960 \mathrm{~s}$, primarily driven by the innovations in military and space sectors, coupled with the expertise of a few individuals who were using readily available commercial equipment. ${ }^{6}$ Examples of early technological milestones in telemedicine include the use of television to facilitate consultations between specialists at a psychiatric institute and general practitioners at a state mental hospital7, and the provision of expert medical advice from a major teaching hospital to an airport medical centre. ${ }^{8}$

The introduction and popularization of internet has further accelerated the pace of telemedicine, which, in turn expanded its scope and now includes various webased applications (e.g. e-mail, teleconsultations and conferences applications) and multimedia approaches (e.g. digital imagery and video). In addition, in a post COVID world, the use of various teleconsultations is on the rise. 


\section{INTRUMENTS REQUIRED}

In most cases, the primary requirement is of having a stable, high speed internet capable of uploading patient record files quickly. The second major requirement is of having an internet enabled device with camera which needs to be used for communication, modern smartphones and laptops are quite capable of doing so. It is desired that the conversation be encrypted and kept confidential. Also, the uploaded files of the patient need to be maintained on a secure and private server.

There is also a need for a person to be well-versed with operation of such devices. Among the illiterate and old aged people, at times, there might a need for an internet and app savvy person to connect the person with the server.

\section{DEFINITION OF TELEMEDICINE}

As per the WHO, telemedicine is defined as the following though this broad description:

"The delivery of health care services, where distance is a critical factor, by all health care professionals using information and communication technologies for the exchange of valid information for diagnosis, treatment and prevention of disease and injuries, research and evaluation, and for the continuing education of health care providers, all in the interests of advancing the health of individuals and their communities". 9

\section{TELEMEDICINE IN EMERGENCY CASES AND TRIAGE}

In a post COVID-word, hospitals need to be everprepared to handle any emergency/ efficiently perform patient triage. This can be achieved only by improving the efficiency of emergency departments, without compromising on patient care; and telemedicine comes as a boon in such instances. It allows for remote and ambulatory monitoring of patients as well as help undergo remote triage and assessment of emergencies including, but not limited to MI, fractures bones, trauma, etc. ${ }^{10}$

The inclusion of automated forward triage systems that use algorithms to categorize patients into risk groups could also be utilized and same time of emergency respondents and physicians.

\section{TELEMEDICINE AND ITS APPLICATION IN LONG-TERM CARE FACILITIES}

As discussed above, the use of smartphones, tablets and other internet devices among seniors remains quite low. A recent study in the year 2019 revealed that only $21 \%$ of the residents in retirement homes used the internet and among those, $13 \%$ used a smartphone, and $5 \%$ used a tablet." It has been reported that internetbased device adoption is associated with the living environment, individual characteristics, functional health, chronological age, education, and technology interest of a person and is directly related to technology adoption among the oldest age groups. ${ }^{12}$

\section{ARTIFICIAL INTELLIGENCE ASSISTED TELEMEDICINE}

The use of innovative data-driven Artificial Intelligence (AI) algorithms shall apply machine learning to large datasets of disease populations and provide accurate results. An important fact is that these models can learn directly from data without any prior statistical modelling, thus producing more objective results while focusing on prediction generalizability for diagnostic purposes on diverse populations. Ever since the COVID-19 pandemic outbreak, AI has been used for international efforts for COVID-19 forecasting, prevention and treatment by using data-driven tools and pooled datasets from across the globe. ${ }^{13,14}$

An added of advantage of the Machine Learning (ML) model is that it incorporates an important analysis that helps develop cost-effective protocols. Such datadriven computational approaches can test for synergistic variable combinations and elimination of redundancy enables more effective diagnosis of patients under the purview of telemedicine. ${ }^{15}$

\section{LEGAL AND PRIVACY ISSUES/RESOLUTIONS}

There are a few legal and privacy issues pertaining to telemedicine and they are as follows: ${ }^{16}$

a). Liability: Despite its numerous advantages, there is the possibility that a patient may perceive this method as inferior because the consulting professional does not perform a hands-on examination and hence would not be able to reach an accurate diagnosis. A major issue including liability is when information 
provided over the telephone is misinterpreted by either the patient or the attending physician. ${ }^{17}$

b). Reimbursement and Licensure: Unfortunately, there have been incidents in which practitioners who were eligible for reimbursement were unhappy with either the payments made, or due to delayed payments made to them. In addition, healthcare professionals are only licensed to practice within certain jurisdictions, and telemedicine requires multiprovince licensure, which might lead to legal issues both for the service provider and the physician. For physicians, applying for applications for practising license in different provinces/states can be lengthy and expensive process.

c). Privacy and Confidentiality: Telemedicine should ensure that privacy, security, and confidentiality of data of patients must be maintained at all times and only authorized users-those who directly involved in the ongoing care and treatment of a patient-and only those having a legal right can approach the systems to servers.18 It is important that nurses and other healthcare professionals need to be mindful of these issues, especially when technicians not bound by professional codes of ethics are present at telemedicine sessions. ${ }^{1}$

d). Quality: Internet speed; and access to information at any time, from any place, are essential to maintaining a high quality of service; slowdowns or outages in service are not acceptable; especially during emergency situations or where immediate triage is necessary. The scientific literature has reported inadequate funds among various establishments for maintenance of the technological infrastructure. ${ }^{19}$

\section{CONCLUSION}

In a post COVID world, there is no doubt that telemedicine will grow manifold and people will enjoy the benefits offered it. However, one needs to be cautious of its drawbacks provide evidence based guidelines on a timely basis for the efficient use of this technology.

\section{REFERENCES}

1. Alvandi M. Telemedicine and its Role in Revolutionizing Healthcare Delivery. The American Journal of Accountable Care. 2017;5(1):e1-e5.

2. Al-Khalifa KS, AlSheikh R. Teledentistry awareness among dental professionals in Saudi Arabia. PLoS ONE
2020;15(10):

https://doi.org/10.1371/journal.pone.0240825

e0240825. 3. Mihailovic B, Miladinovic M, Vujicic B. Telemedicine in Den tistry (Teledentistry). In: Graschew G and Roelofs TA(editors). Advances in Telemedicine: Applications in Various Medical Disciplines and Geographical Areas 2011. Rijeka (Croatia): InTech, 2011. p. 215-30.

4. Jampani ND, Nutalapati R, Dontula B, Boyapati R. Applications of teledentistry: A literature review and update. J Int Soc Prevent Communit Dent 2011;1:37-44. 5. Craig J, Patterson V. Introduction to the practice of telemedicine. Journal of Telemedicine and Telecare, 2005, 11(1):3-9.

6. Currell $\mathrm{R}$ et al. Telemedicine versus face to face patient care: effects on professional practice and health care outcomes. Cochrane Database of Systematic Reviews, 2000, Issue 2. Art. No.: CDoo2098.

7. Benschoter RA, Eaton MT, Smith P. Use of videotape to provide individual instruction in techniques of psychotherapy. Academic Medicine, 1965, 40(12):115961.

8. Dwyer TF. Telepsychiatry: psychiatric consultation by interactive television. American Journal of Psychiatry, 1973, 130:865-9.

9. WHO. A health telematics policy in support of WHO's Health-For-All strategy for global health development: report of the WHO group consultation on health telematics, 11-16 December, Geneva, 1997. Geneva, World Health Organization, 1998.

10. Badawy SM, Radovic A. Digital approaches to remote pediatric health care delivery during the COVID-19 pandemic: existing evidence and a call for further research. JMIR Pediatr Parent. (2020) 3:e20049. https://doi.org/10.2196/20049

11. Seifert A, Cotten SR. In care and digitally savvy? Modern ICT use in long-term care institutions. Educ Gerontol. (2020). 46:473-85. https://doi.org/10.108o/03601277.2020.1776911

12. Schlomann A, Seifert A, Zank S, Rietz C. Assistive technology and mobile ICT usage among oldest-old cohorts: comparison of the oldest-old in private homes and in long-term care facilities. Res Aging. (2020) 42:163-73. https://doi.org/10.1177/0164027520911286 13. Huys QJM, Maia TV, Frank MJ. Computational psychiatry as a bridge from neuroscience to clinical applications. Nat Neurosci. (2016) 19:404-13.

https://doi.org/10.1038/nn.4238

14. Kuziemsky C, Maeder AJ, John O, Gogia SB, Basu A, Meher S, et al. Role of artificial intelligence within the telehealth domain. Yearb Med Inform. (2019) 28:35-40. https://doi.org/10.1055/s-0039-1677897 
15. Pacis DMM, Subido EDC, Bugtai NT. Trends in telemedicine utilizing artificial intelligence. AIP Conf Proc. (2018) 1933:040009. https://doi.org/10.1063/1.5023979 16. Dickens BM, Cook RJ. Legal and ethical issues in telemedicine and robotics. Int J Gynaecol Obstet. 2006;94(1):73-8.

Source of support: Nil, Conflict of interest: None declared
17. Hebda TL, Czar P. Handbook of Informatics for Nurses \& Healthcare Professionals. 4th ed. Upper Saddle River, NJ: Pearson Prentice Hall; 2009.

18. McGonigle D, Mastrian KG. Nursing Informatics and the Foundation of Knowledge. 2nd ed. Burlington, MA: Jones and Bartlett Learning; 2011

19. Bond GE. Lessons learned from the implementation of a Webbased nursing intervention. Comput Inform Nurs. 2006;24(2):66-74.
Cite this article as:

Sharma B, Manocha S, Yangzom N. The Importance of Telemedicine in a Post COVID World. Int Healthc Res J. 2021;5(7):RV11-RV14. https://doi.org/10.26440/IHRJ/0507.10467 